Please do not remove this page

RMIT

UNIVERSITY

\title{
Nanolithography by elastomeric scattering mask: An application of photolithographic standing waves
}

Kostovski, Gorgi; Mitchell, Arnan; Holland, Anthony; Fardin, Ernest; Austin, Michael

https://researchrepository.rmit.edu.au/esploro/outputs/9921860091501341/filesAndLinks?institution=61RMIT_INST\&index=null

Kostovski, G., Mitchell, A., Holland, A., Fardin, E., \& Austin, M. (2006). Nanolithography by elastomeric scattering mask: An application of photolithographic standing waves. Applied Physics Letters, 88(13), 1-3. https://doi.org/10.1063/1.2190899

Published Version: https://doi.org/10.1063/1.2190899

Repository homepage: https://researchrepository.rmit.edu.au

(c) 2006 American Institute of Physics. This article may be downloaded for personal use only. Any other use requires prior permission of the author and the American Institute of Physics.

Downloaded On 2023/04/26 22:55:25 +1000 


\title{
Nanolithography by elastomeric scattering mask: An application of photolithographic standing waves
}

\author{
Gorgi Kostovski, ${ }^{\text {a) }}$ Arnan Mitchell, Anthony Holland, Ernest Fardin, and Michael Austin \\ Microelectronics and Materials Technology Centre (MMTC), School of Electrical and Computer \\ Engineering, RMIT University, G.P.O. Box 2476, Melbourne, Victoria 3001, Australia
}

(Received 9 November 2005; accepted 15 February 2006; published online 31 March 2006)

\begin{abstract}
An application is demonstrated for the much maligned standing wave in photolithography that is responsible for the sidewall corrugations in photoresist patterns. We demonstrate the realization of a polydimethylsiloxane (PDMS) scattering mask through the casting of these sidewall corrugations and their application as the masking components in an otherwise transparent bulk of PDMS. Photoresist structures with widths in the order of $80 \mathrm{~nm}$ are realized by the application of this mask, demonstrating excellent correlation with the lateral depths of the sidewall corrugations. The continuity of the sidewall corrugations around corners makes both straight and curved lines realizable with equal simplicity. (c) 2006 American Institute of Physics. [DOI: 10.1063/1.2190899]
\end{abstract}

Contact photolithography has long struggled with the realization of nanometer scale patterning of photoresists due to difficulties in achieving conformal contact between its rigid masks and the photoresist surface. More sophisticated photolithographic techniques, including deep UV, electron beam lithography, and focused ion beam lithography have shown excellent results, although limited access to costly infrastructure has restricted their application. Elastomeric masks, however, as pioneered by Whitesides and co-workers, ${ }^{1,2}$ have proven themselves to be more than capable of realizing nanometer scale structures in photoresist. These masks are developed and applied using standard photolithographic techniques and equipment.

Simple implementations such as the light coupling ${ }^{3}$ and transparent reflective masks ${ }^{1}$ have demonstrated microscale patterning in both 1:1 and 2:1 pattern density ratios by the mechanisms of differential transmission and total internal reflection, respectively. More recently, the elastomeric phase mask has demonstrated both 90 (Ref. 2), and $50 \mathrm{~nm}$ (Ref. 4) resist features by using the recessed regions of the elastomeric mask to induce $\Pi$ radian phase shifts between regions of transmitted light, thus realizing intensity nulls beneath the sidewalls of the mask features.

Traditionally elastomeric masks have been realized by casting master photoresist structures patterned by standard lithography. Unfortunately, lithography has long been troubled by a sidewall roughness that is not defined by molecular resolution. Rather, this sidewall roughness is caused by a periodic variation in light intensity through the resist that is the result of interference between the normally incident planar wave front and that reflected from the substrate/ resist interface. The result is a periodic corrugation of the resist sidewalls that is oriented perpendicular to the surface normal. Much effort has been expended in eliminating these corrugations. ${ }^{5}$ The development of top and bottom antireflection coatings, the employment of postexposure bakes, and the inclusion of attenuating dyes in the photoresist are only some of the avenues that have been pursued.

In this letter, we present a novel implementation of polydimethylsiloxane (PDMS) elastomer mask that is capable of

\footnotetext{
a)Electronic mail: s9909628@ student.rmit.edu.au
}

realizing nanometer scale features comparable to those demonstrated by the elastomeric phase mask, ${ }^{2,4}$ but which operates by a significantly different mechanism. We demonstrate that the mechanical flexibility of PDMS makes it uniquely suited to taking faithful casts of these lateral relief structures in photoresist, as has been demonstrated elsewhere on a much larger scale. ${ }^{6}$ This allows us to exploit these corrugations as the mechanism by which the PDMS scattering mask operates. Thus, just as sloped sidewalls are required for the reflection mask to realize total internal reflection, and smooth vertical walls are required for the elastomeric phase mask to allow spatial overlap between neighboring out of phase wave fronts, the sidewall corrugations that appear in photolithography become the masking elements in this new implementation of elastomeric mask.

The procedure employed to generate the nanoscale structures presented in this letter is illustrated in Fig. 1. Master fabrication begins by depositing an $\sim 200 \mathrm{~nm}$ thin film of the positive photoresist AZ5206E onto a polished silicon wafer by closed lid spinning at $3300 \mathrm{rpm}$ for $30 \mathrm{~s}$. This is patterned by contact photolithography using a quartz mask and a broadband Karl Suss MJB3 mask aligner. Developing the pattern realizes rectangular ribs of photoresist with prominent sidewall corrugations, shown in Figs. 2(a)-2(c). A cast is then taken of this master structure by applying a 10:1 mixture of PDMS (Dow Corning 184 Silicone Elastomer Kit), curing it, and then separating it from the photoresist pattern to produce a thick $5 \mathrm{~mm}$ sheet of PDMS with surface recesses. These recesses correspond to the master photoresist ribs, on the sidewalls of which are the standing wave corrugations. This is the PDMS scattering mask.

The scattering mask is applied by allowing it to conform to the surface of a fresh layer of AZ5206E photoresist and illuminating it with the same mask aligner that was used to create the master pattern. The scattering mask presents the normally incident planar wave front with three distinct paths to the resist below it, illuminating the photoresist with three distinct energies as in Fig. 1(f). Maximum energy is coupled by the PDMS that contacts the photoresist surface. Slightly less energy is received by photoresist directly below the PDMS recesses since incident light is presented with a PDMS/air and then air/photoresist interface. This energy dif- 
a)

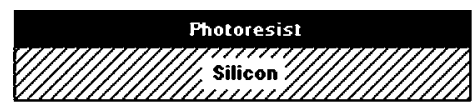

b)

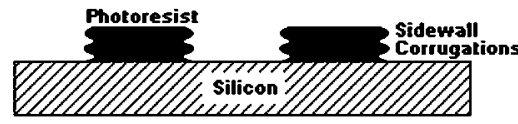

c)

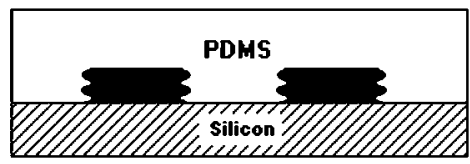

d)

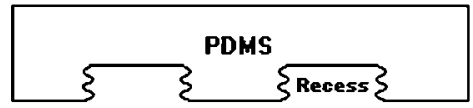

e)

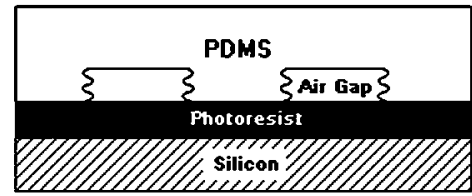

f)

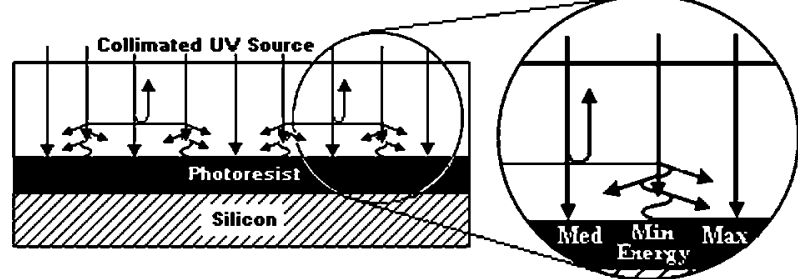

g)

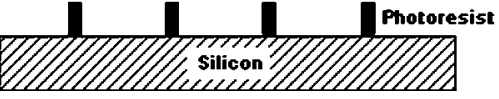

FIG. 1. The fabrication and application of a PDMS scattering mask. (a) A master structure is realized by spinning a layer of photoresist onto a reflective substrate, in this case a polished silicon wafer, which is (b) photolithographically patterned using a quartz mask. (c) Casting PDMS against this structure realizes (d) the PDMS scattering mask. (e) This mask is applied by conforming it to the surface of a new layer of photoresist and (f) illuminating the photoresist through the scattering mask. (g) Developing realizes nanometer linewidths.

ference is the mechanism by which the light coupling mask operates. Finally, minimum energy is received by photoresist directly below the corrugated sidewalls since the wave front is presented with multiple vertically stacked PDMS/air and air/PDMS interfaces. The light that intercepts these sidewall corrugations is scattered and largely prevented from entering the photoresist. Thus the sidewall corrugations are employed as the dominant masking components in an otherwise relatively transparent bulk of PDMS. This distribution of energies has been verified by intentionally underdeveloping photoresist that has been exposed by the scattering mask to observe its progressive development. Photoresist residue is clearly observed in regions that correspond to recesses in the scattering mask, while photoresist that made contact with the PDMS was fully developed.

Completely developing the exposed photoresist realizes the $\sim 80 \mathrm{~nm}$ wide lines in Figs. 3(a)-3(c) whose positions correspond to the sidewalls of each of the master photoresist ribs. Ironically, we see some broadening of our linewidth from $\sim 80$ to $\sim 100 \mathrm{~nm}$ at the center of the rib as a result of standing waves. The realized features are continuous and, since they were realized in a $200 \mathrm{~nm}$ thick layer of resist, demonstrate an aspect ratio of 2.5:1. Aspect ratios of 10:1 have been realized using a scattering mask with $800 \mathrm{~nm}$ deep recesses, although these features exhibit significant broadenDownloaded 19 Nov 2008 to 131.170.90.4. Redistribution subject
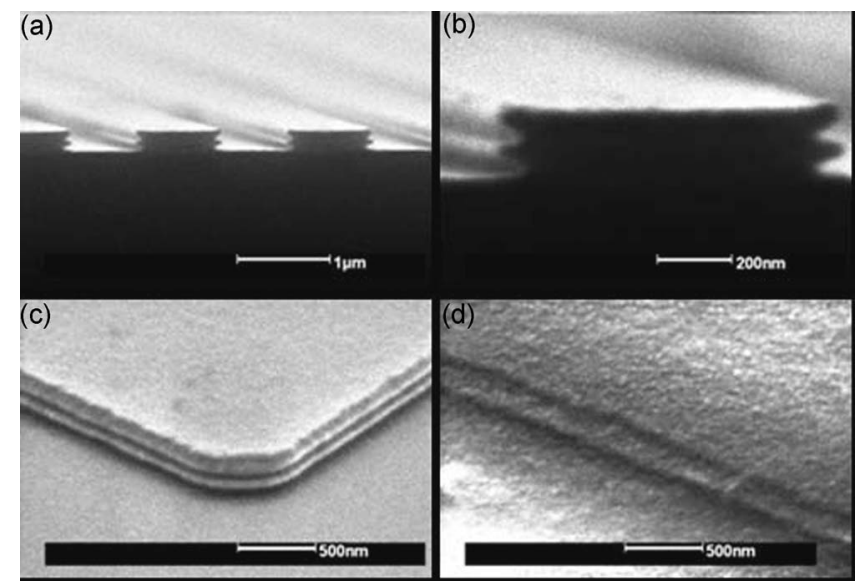

FIG. 2. Scanning electron microscope images of the master photoresist structures used to realize the PDMS scattering mask. (a) The regular depth and vertical stacking of the corrugations over several lines is shown clearly in broad view cross section, while (b) a higher magnification reveals the $\sim 80 \mathrm{~nm}$ depth of the sidewall corrugations. (c) An illustration of the continuity of the corrugations around corners as well as the independence of the corrugations on the photoresist thickness. (d) The sidewall corrugations in photoresist are clearly transferred to PDMS, realizing the PDMS scattering mask.

ing in cross section as they approach the substrate. An $80 \mathrm{~nm}$ feature width has been shown to taper to $200 \mathrm{~nm}$, over a height of $800 \mathrm{~nm}$.

As a potential application of these photoresist features, we have demonstrated the patterning of a $50 \mathrm{~nm}$ layer of gold by lift-off, shown in Figs. 3(d)-3(f). Excellent correlation is again demonstrated between the width of the gold trenches and the depths of the sidewall corrugations in the

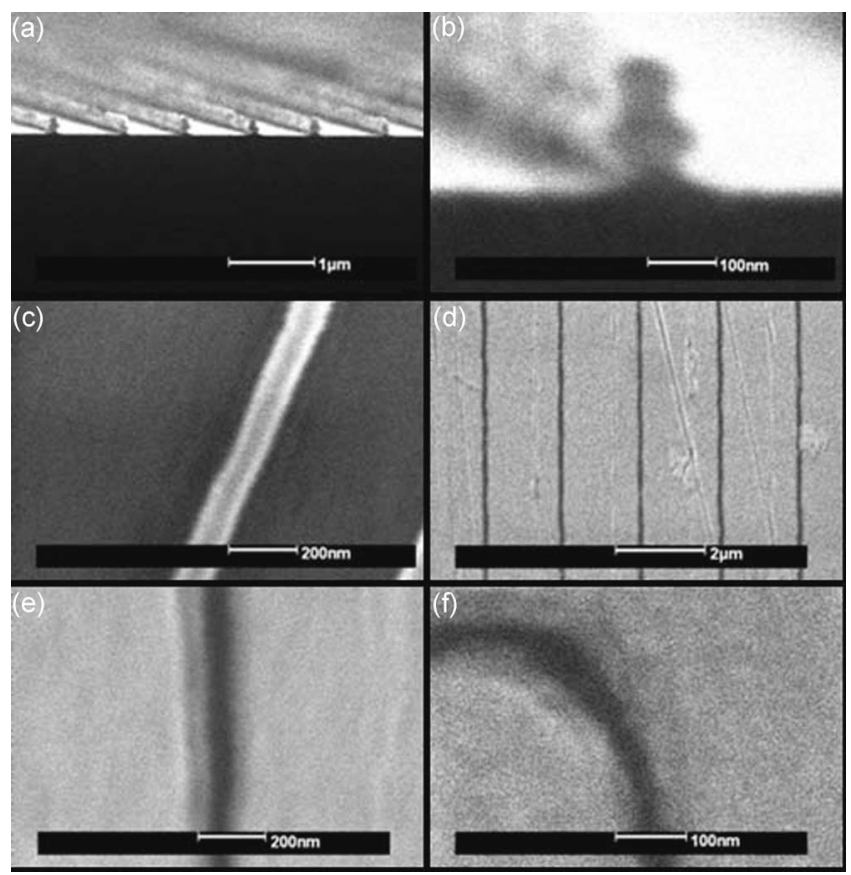

FIG. 3. Scanning electron microscope images of the structures realized by applying the PDMS scattering mask. (a) The regularity of the $\sim 80 \mathrm{~nm}$ lines of positive photoresist over a large area is shown in broad cross section, while (b) a closer examination reveals the $\sim 80 \mathrm{~nm}$ lateral dimensions of the ribs, in excellent correlation with the depth of the master sidewall corrugations. (c) A top down view. (d) Lift-off generates trenches in a $50 \mathrm{~nm}$ thick layer of gold. (e) A closer view reveals a linewidth in agreement with the photoresist lines as well as (f) continuity around corners.

AIP license or copyright; see http://apl.aip.org/apl/copyright.jsp 
master pattern. We also see continuity of the trenches around corners in Fig. 3(f), confirming pattern transfer from Fig. 2(c). The narrower than expected linewidth in this curved line is attributed to overdeveloping.

Feature dimensions produced by this mask are defined by how well the sidewall corrugations are confined horizontally, as well as the depth of the corrugations themselves. For the photoresist used in this case, it was found that vertically stacked corrugations and thus narrower features were more easily realized with a thinner film of photoresist. However, the specific thickness employed to fabricate the photoresist master pattern is not critical. A comparison of the sidewall corrugations in Fig. 2(b) and those in the slightly thicker layer of photoresist in Fig. 2(c) demonstrates that the presence of the standing wave corrugations is independent of photoresist thickness. The downward reflections of the vertically traveling wave front from the photoresist/air interface are too weak to significantly dilute the standing wave interference pattern generated by the normally incident and substrate reflected wave fronts. As a result, the master patterns in Figs. 2(b) and 2(c) provide equivalent performance when applied as scattering masks. A significant advantage of this fact is that it makes the scattering mask technique insensitive to processing tolerances that may lead to variations in photoresist thickness, both across batches and, to a lesser degree, across single wafers.

For the purpose of eliminating destructive interference as the masking mechanism in the scattering mask, consider that the phase shifts induced by the 200 and $800 \mathrm{~nm}$ recesses implemented here have been calculated to be $2 \Pi / 5$ and $2 \Pi$ radians, respectively, effecting constructive interference. This is in stark contrast with the $\Pi$ phase shift induced by the $400 \mathrm{~nm}$ recesses in the elastomeric phase mask. ${ }^{2,4}$

Some simple limitations on master fabrication are set by the elastomeric properties of the mold material and apply to all variations of elastomeric mask. Broad ribs on the master will translate to broad recessed regions which are subject to sagging. This sagging may bring the ceiling of the recess into contact with the photoresist if the recess width-to-height ratio is too great, deforming the recess sidewalls. This begins to occur at $7 \mu \mathrm{m}$ linewidths for a $200 \mathrm{~nm}$ thick resist with a 10:1 PDMS mix. The authors speculate that features with separations narrower than those demonstrated here may be achievable if master structures are realized holographically, as for Bragg grating fabrication, rather than using solid quartz masks. Of course, the masking mechanism of scattering may impose proximity limitations on features realizable with this technique due to scattered light illuminating neigh- boring masked regions. Conversely, casting structures with widths in the order of nanometers will see neighboring features in the mold being drawn together if the aspect ratios on the master structures are too high.

Although this technique is limited to realizing a single feature width across any one mask, it is likely that this width can be tuned over a wide range of feature dimensions by using different photoresists and processing techniques. To this end, we note that sidewall corrugations in literature have been observed with depths as shallow as $\sim 11 \mathrm{~nm}^{7}$

In closing, we have demonstrated a lithographic technique that realizes $\sim 80 \mathrm{~nm}$ features in photoresist from micron sized features on quartz masks. This has been achieved through the exploitation of a feature of photolithography that has until now been consistently targeted for elimination. The technique is insensitive to variations in thickness of the master pattern, it makes use of a feature that would otherwise require additional steps to eliminate it, and it is realized by using standard contact photolithographic equipment. Immediate applications include period halving of periodic structures and realizing either very narrow features or very close proximity structures. The applications of this technique can be extended beyond those demonstrated here, by employing standard variations in photolithographic procedure, such as using negative photoresist or executing consecutive orthogonal exposures, to realize both trench and post features in photoresist, respectively.

In a broader context, we have demonstrated that the technique of PDMS casting is well suited to undercut structures. These are structures that are intrinsically impossible to replicate with techniques that use rigid mold materials. Work is continuing to realize features even narrower than the $80 \mathrm{~nm}$ demonstrated here.

${ }^{1}$ D. Quin, Y. Xia, A. J. Black, and G. M. Whitesides, J. Vac. Sci. Technol. B 16, 98 (1998).

${ }^{2}$ J. A. Rogers, K. E. Paul, R. J. Jackman, and G. M. Whitesides, J. Vac. Sci. Technol. B 16, 59 (1998).

${ }^{3}$ H. Schmid, H. Biebuyck, and B. Michel, Appl. Phys. Lett. 72, 2379 (1998)

${ }^{4}$ J. Maria, S. Jeon, and J. A. Rogers, J. Photochem. Photobiol., A 166, 149 (2004).

${ }^{5}$ A. G. Grandpierre, R. Schiwon, and F. Finger, Metrology, Inspection, and Process Control for Microlithography XIX, edited by M. Voet, R. Willsch, W. Ecke, J. Jones, and B. Culshaw (SPIE, Bellingham, WA, 2005), p. 755.

${ }^{6}$ N. Bogdanski, H. Schulz, M. Wissen, H.-C. Scheer, J. Zajadacz, and K. Zimmer, Microelectron. Eng. 73-74, 190 (2004).

${ }^{7}$ A. K. Bates, M. Rothschild, T. M. Bloomstein, T. H. Fedynyshyn, R. R. Kunz, V. Liberman, and M. Switkes, IBM J. Res. Dev. 45, 605 (2001). 